\title{
3-Dimensional Activation Sequence Reconstruction from Body Surface Potential Maps by Means of a Heart-Model-Based Imaging Approach
}

\author{
X Zhang ${ }^{1}$, I Ramachandra ${ }^{2}$, Z Liu $^{1}$, B Muneer ${ }^{2}$, \\ SM Pogwizd ${ }^{2}, \mathrm{~B} \mathrm{He}^{1}$ \\ ${ }^{1}$ University of Minnesota, Minneapolis, MN, USA \\ ${ }^{2}$ University of Illinois at Chicago, Illinois, IL, USA
}

\begin{abstract}
In the present study, a heart-model-based imaging approach was applied to the rabbit heart, and 3-D cardiac activation sequences were estimated noninvasively from body surface potential maps during in vivo ventricular pacing from different sites. The estimated activation sequences exhibited earliest sites of cardiac activation and patterns of activation that were consistent with those constructed from 3-D activation sequences obtained from simultaneously recorded bipolar electrogram data (from nearly 160 intramural recording sites). The relative errors between the imaged and measured activation sequences were $0.291 \pm 0.009$ for the cases studied. The present study suggests that the heart-model-based imaging approach represents an important alternative for noninvasive imaging of cardiac activation that could be useful for basic cardiovascular research and for the diagnosis and management of cardiac diseases.
\end{abstract}

\section{Introduction}

Cardiac activation imaging throughout the three dimensional (3D) myocardium can provide useful information for basic cardiovascular research and for diagnosis and management of cardiac diseases. In the past decades, a tremendous amount of effort has been made to noninvasively obtain information on cardiac activation from the body surface potential map (BSPM). Most of such efforts have been in attempting to image the activation sequence over the heart surface from the BSPM [1-5]. Such approaches are limited in that it only provides activation sequence over the $2 \mathrm{D}$ heart surface.

Recently, several efforts have been made to attempt to image 3D cardiac electrical activity from the BSPM. A Laplacian-weighted minimum norm (LWMN) algorithm with a recursive weighting strategy was used to obtain the 3D source distribution within myocardium from the BSPM [6,7]. A noninvasive 3D cardiac activation imaging approach was recently proposed by means of a heart-excitation-model and tested by computer simulation studies $[8,9]$. A similar effort was also reported using a ventricular excitation model in the MCG inverse problem, and a priori information about activation sequence and action potentials were used in combination of a nonlinear optimization procedure [10]. Furthermore, estimation of cardiac transmembrane potential distributions have also been reported, using the heart-modelbased approach $[8,11]$, the Laplacian weighted minimumnorm algorithm [12], or the inverse Wiener filter [10].

The aim of the present study was to experimentally validate the heart-model-based activation time imaging approach [8, 9] using a rabbit model [13-15]. The noninvasively estimated activation sequences were compared with myocardial activation sequences measured within the 3D myocardium of the rabbits using an array of inserted electrodes. We report, for the first time, experimental evidence to demonstrate the feasibility of noninvasively imaging 3-D activation sequences throughout the myocardium from the BSPM, an approach that may become a useful means for cardiovascular research and clinic diagnosis of cardiac diseases.

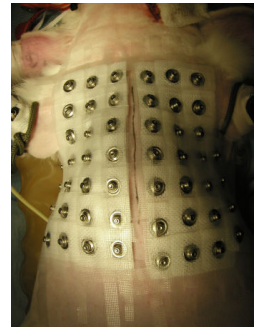

(a)

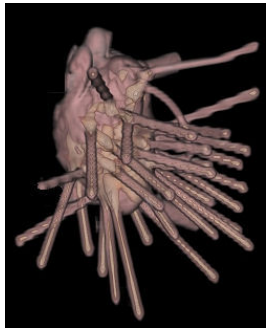

(b)
Fig. 1 (a) Body surface electrode placement. (b) A 3D reconstruction from ultrafast-CT scan images of an explanted rabbit heart with labeled pins replacing the plunge needle electrodes used during intraoperative 3D mapping

\section{Methods}

\subsection{Animal model and in vivo mapping}

Healthy New Zealand rabbits were studied using a protocol 
approved by the Institute Review Board of the University of Minnesota and the University of Illinois at Chicago. The anatomical geometry information was obtained by Ultra Fast CT (UFCT) one day before the in vivo mapping experimentation. Two sets of CT images were obtained with ECG gating at the peak of the QRS complex. The first set of images, a continuous volume scan with slice thickness of $3 \mathrm{~mm}$ from the level of the mid neck down to the lower abdomen, was used to construct the rabbit torso model. The second set of images, from the atria or great vessel level down to the diaphragm, had a slice thickness of $3 \mathrm{~mm}$ with $2 \mathrm{~mm}$ overlap. IV contrast was administered to obtain the ventricular endocardial chamber geometry. The respiration of rabbit was held during the scan in order to minimize respiratory artifact. The detailed ventricular model was built afterwards based on this set of CT images.

Simultaneous body surface potential mapping and 3-D bipolar mapping of cardiac electrical activity were conducted as described below. The torso and limbs of rabbit were shaved and the skin was carefully prepared for placement of surface electrodes. 48 breathable electrodes for infants (Vermed, VT) were placed uniformly to cover the anterolateral chest up to the mid-axillary line (Fig. 1(a)). The electrode locations and fiducial points, as well as the torso geometry, were digitized 3 times using a radio frequency localizer (Polhemus Fastrak. VT), which provides a subjectspecific coordinate reference for registering electrode positions with the geometry torso model based on the UFCT scans. Then the heart was exposed via median sternotomy, and 20 transmural needle electrodes were inserted in the left and right ventricles of the rabbit (Fig. 1(b)). Each needle electrode contains 8 bipolar electrode-pairs with an interelectrode distance of $500 \mu \mathrm{m}$ (160 total intramural sites). The chest was then closed and rapid ventricular pacing (basic cycle length of 180-100 msecs, 10-30 second drive trains) was performed via bipolar electrode pairs on selected needle electrodes, while 3-D transmural and transseptal ventricular activations were continuously recorded from all 160 bipolar electrode pairs together with body surface potentials from 48 surface electrodes. Bipolar electrogram data were acquired at $1 \mathrm{kHz}$, A-to-D converted (using a multichannel cardiac mapping system (Crescent Electronics, Salt Lake City). Digital data were stored on an Apple G4 computer using specially-designed software . Simultaneous body surface potentials were simultaneously acquired during ventricular pacing at a sampling rate of $1 \mathrm{kHz}$ (NeuroScan, TX). After all pacing was completed, spatial localization of body surface electrodes and torso shape were re-digitized. Prior to excision of the heart, the plunge needle electrodes were carefully localized by replacing each with a labeled pin. The heart was then excised and fixed in formlin. At a later date, the formalin-fixed heart with labeled pins was scanned in the UFCT to get precise 3D localization of the transmural electrodes.

\subsection{Heart-model-based imaging}

A 3-D heart-excitation-model was constructed for each of the rabbits studied from geometric measurements via UFCT. A preliminary classification system (PCS) which approximately determines cardiac status based on a priori knowledge and the measured BSPMs was used to estimate the initial pattern of myocardial activation by means of an artificial neural network (ANN) $[9,16]$. According to the output of the PCS, the parameters of the heart model are initialized, and the corresponding BSPM was calculated using the boundary element method (BEM). Then a multiobjective nonlinear optimization procedure was employed to estimate the cardiac activation sequence. If the measured and model-generated BSPMs match well, the optimization procedure stopped. If not, the heart model parameters were adjusted with the aid of the optimization algorithms and the simulation procedure proceeds until the objective functions satisfied the given convergent criteria. The activation sequence produced by the optimized heart model parameters were compared with the measured activation sequence (see below) to assess the performance of the noninvasive 3D myocardial activation imaging. Fig. 2 illustrates a schematic

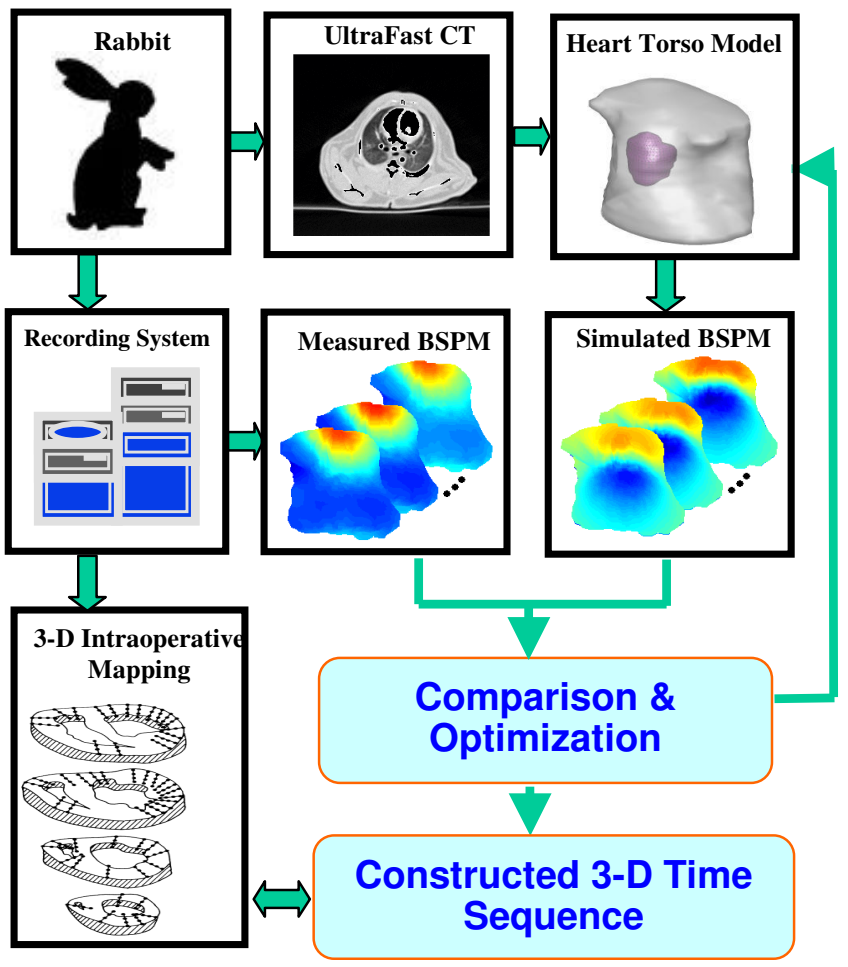

Fig. 2 Schematic diagram of the experimental validation of the heart-model-based activation imaging approach

diagram of this experimental validation.

\subsection{3-D intraoperative mapping}

The electrograms recorded from transmural needle electrodes were analyzed with an automated system [13-15]. 
Computer-assigned activation sequences were based on a peak criterion. Review and editing of electrograms were

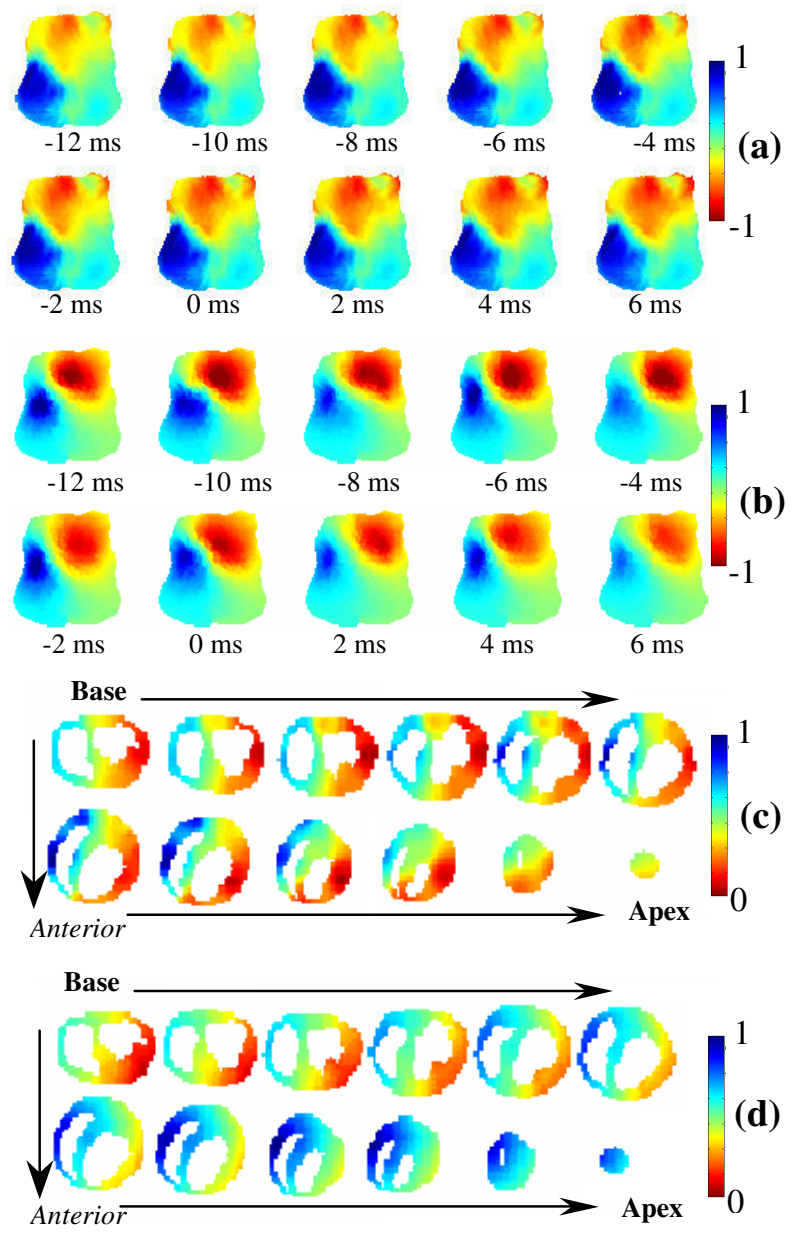

Fig. 3 Pacing from right ventricle apex: (a) Measured BSPMs; (b) Inversely estimated BSPMs bestfitting the measured BSPMs shown in (a); (c) Measured 3-D activation sequence throughout the myocardium; (d) Inversely estimated 3-D activation sequence from the model-based imaging approach.

performed by the operator as previously described [13-15]. The 3-dimentional activation maps were obtained as following: First, the precise coordinates of all bipolar electrode pairs were decided from the 3-D isolated heart model with each transmural needle electrode (using a software package developed in our group). Then, based on the anatomical and pathological information, a $3^{\text {rd }}$ order polynomial transformation [17] was used to best-fit the epicardial surface of isolated heart to the epicardial surface of heart model used in our imaging study. The coordinates of bipolar electrode pairs could also be mapped to this heart model. Small adjustment of best-fit parameters were applied to ensure both the surface fit and linearity of transformed electrode position. The corresponding activation sequence of each bipolar electrode pair were then assigned, upon which a weighted average interpolation algorithm [18] was applied to get the complete 3-D activation sequence map throughout the myocardium. The overall pattern of activation sequences, as well as origin of activation can be obtained from this set of 3-D activation sequence maps, which was used to evaluate our 3-D activation sequence imaging results in multiple sections (Fig. 3).

\section{Results}

Stimulation of the heart involved bipolar endocardial or epicardial pacing via selected transmural needle electrodes that were inserted throughout the left and right ventricles. The sequential pace maps were obtained by solving the forward problem. 194-lead (uniformly distributed over the anterior-lateral torso surface) BSPMs were calculated in each pacing sequence within the whole cardiac excitation cycle using the boundary element method. The time points of which the BSPMs used in the optimization procedure were chosen at around the simulated peak activity on a surface electrode located at mid-anterior-left torso. Usually we chose the electrode that provided the best signal to noise ratio. For the measurement of BSPMs, the surface electrodes were registered to the heart-torso model based on the fiducial points (as well as digitized torso shape points) by a surfacematching algorithm [19]. The time points of measured BSPMs were taken similarly as simulated BSPMs. A SPLINE interpolation algorithm was used to interpolate the measured BSPMs at 194-sites from 48 surface electrodes' measurement.

Fig 3 is an example in which the pacing stimulation initiated at the apical right ventricle and propagated both leftward and basally. Fig 3 (a) shows the measured BSPMs, with a time interval of $2 \mathrm{~ms}$. It shows a dipolar pattern over the anterior torso surface with positive activity at upper-left and negative activity at lower-right. Fig 3 (b) shows the corresponding simulated BSPMs that were the best-match above measured BSPMs (after the optimization of our imaging procedure). Similar dipolar pattern is evident, although estimated BSPMs are smoother and more focal compared to measured ones. Fig 3 (c) shows the measured 3$\mathrm{D}$ activation sequence from ventricular base to ventricular apex derived from intracardiac electrograms of the same beat as in Figs 3a \& 3b. Earliest activation (blue area) initiated in the mid-right ventricle just slightly closer to apex (second slice on second row), which then propagated to the left ventricle. This result was confirmed by pinpointing the transmural electrode on which the stimulation was induced, and the pattern of activation sequence was similar with referred hand-drawn activation maps over the templates of rabbit heart slices. Fig 3 (d) shows the estimated 3-D activation sequence obtained by our imaging approach, which also revealed early activation in the right ventricle, but just slightly more towards apex (third slice on second row). The quantitative comparison between Fig. 3 (c) and (d) gives a relative error $\mathrm{RE}=0.285$. Three more pacing beats 
from additional pacing location were analyzed to get statistical results, which gives $\mathrm{RE}=0.291 \pm 0.009$.

\section{Discussion and conclusions}

In present study, 3-D activation sequences have been reconstructed from noninvasive BSPMs, by means of a heart-model-based imaging approach. Quantitative comparison to measured intracardiac activation patterns allows to evaluate the performance of the estimated activation sequence within 3-D myocardium. The pilot results are highly promising and show a high level of consistency. Applying a more robust (complicated) heart model is expected to yield even better results as well as a better understanding of rabbit heart activation mechanisms. These promising results suggest that this approach may become an important alternative for noninvasive imaging of cardiac activation for both basic cardiovascular research and clinical diagnosis and management of cardiac diseases.

\section{Acknowledgements}

The authors wish to thank J. Jeanes, T. Dahl, H. Mansoor, Dr. J. Lian, Y. Ni, and B. Muddasir for technical assistance. The authors also wish to thank V. Jelnin for the acquisition of Ultra Fast CT scan. This work was supported in part by NSF BES-0201939, American Heart Association Grant 0140132N, NIH R01EB00178, and NIH R01HL-46929.

\section{References}

[1] Cuppen JJM and van Oosterom A, Model studies with inversely calculated isochrones of ventricular depolarization. IEEE Trans. Biomed. Eng., 1984:31: 652-659.

[2] Huiskamp G, Simulation of depolarization in a membrane-equations-based model of the anisotropic ventricle. IEEE Trans. Biomed. Eng., 1998:45:847-855

[3] Greensite F, "Myocardial Activation Imaging". In: Computational Inverse Problems in Electrocardiography, edited by P. Johnston. WIT press, Brisol, 2001: 143-190.

[4] Modre R, Tilg B, Fischer G, and Wach P. An iterative algorithm for myocardial activation time imaging. Computer Methods and Programs in Biomedicine 2001: 64: 1-7.

[5] Pullan AJ, Cheng LK, Nash MP, Bradley CP, and Paterson DJ. Noninvasive electrical imaging of the heart: theory and model development. Ann Biomed Eng. 2001: 29: 817-836.

[6] $\mathrm{He} \mathrm{B}, \mathrm{Wu} \mathrm{D}$, Three-dimensional source imaging of cardiac electric activity. Prof. of World Congress on Medical Physics and Biomedical Engineering, CDROM, 2000.

[7] $\mathrm{He} \mathrm{B}, \mathrm{Wu} \mathrm{D}$, Imaging and visualization of 3-D cardiac electric activity. IEEE Trans. Inf. Technol. Biomed. 2001: 5:181-186.

[8] He B, Li G, Noninvasive three-dimensional myocardial activation time imaging by means of a heart-excitation- model. Int. J. of Bioelectromagnetism, 2002: 4(2): 8788.

[9] He B, Li G, Zhang X, Noninvasive three-dimensional activation time imaging of ventricular excitation by means of a heart-excitation model. Phys. Med. Biol., 2002: 47: 4063-4078.

[10] Ohyu S, Okamoto Y, and Kuriki S, Use of ventricular propagated excitation model in the magnetocardiographic inverse problem for reconstruction of electrophysiological properties. IEEE. Trans. Biomed. Eng. 2002: 49: 509-519.

[11] He B, Li G, Zhang $\mathrm{X}$, Noninvasive Imaging of Ventricular Transmembrane Potentials within Threedimensional Myocardium by Means of a Realistic Geometry Anisotropic Heart Model. IEEE Trans. Biomed. Eng., 2003: 50: 1190-1202.

[12] Skipa O, Sachse NF, Werner C, Dossel O, Transmembrane potential reconstruction in anisotropic heart model. Proc. of International Conference on Bioelectromagnetism, 2002: 17-18.

[13] Pogwizd SM, Hoyt RH, Saffitz JE, Corr PB, Cox JL, Cain ME. Reentrant and focal mechanisms underlying ventricular tachycardia in the human heart. Circulation. 1992: 86: 1872-1887.

[14] Pogwizd SM, Chung MK, Cain ME. Termination of ventricular tachycardia in the human heart: insights from three-dimensional mapping of nonsustained and sustained ventricular tachycardia. Circulation. 1997: 95: 2528-2540.

[15] Chung MK, Pogwizd SM, Miller DP, Cain ME. Threedimensional mapping of the initiation of nonsustained ventricular tachycardia in the human heart. Circulation. 1997: 95: 2517-2527.

[16] $\mathrm{Li} \mathrm{G}$ and He B, Localization of the site of origin of cardiac activation by means of a heart-model-based electrocardiographic imaging approach, IEEE Trans. Biomed. Eng., 2001: 48: 660-669.

[17] Castleman KR, Digital Image Processing, Prentice-Hall International, Inc. 1996

[18] Dave W, Contouring - A Guide To The Analysis and Display of Spatial Data, Pergamon Press, 1992. ISBN 0-08-040286-0

[19] Towle VL, Bolanos J, Suarez D, Tan K, Grzesczcuk R, Levin DN, Cakmur R, Frank SA, and Spire JP, The spatiallocation of EEG electrodes: Locating the bestfitting sphere relative to cortical anatomy. Electroenceph. Clin. Neurophysiol. 1993: 86: 1-6

Address for correspondence:

Prof. Bin $\mathrm{He}$

7-105 BSBE

312 Church Str.

Minneapolis, MN, 55455

binhe@umn.edu 